\title{
Significance of Laparoscopic Partial Adrenalectomy in the Era of Organ Preservation
}

\author{
Young Beom Jeong Yu Seob Shin Hyung Jin Kim \\ Department of Urology, Jeonbuk National University Medical School, Research Institute of Clinical Medicine \\ of Jeonbuk National University-Biomedical Research Institute of Jeonbuk National University Hospital, \\ Jeonju, Republic of Korea
}

\section{Dear Editor,}

We read with interest the article by Balci et al. [1] regarding the surgical outcomes and clinical improvement 1 year after laparoscopic partial adrenalectomy (LPA) for the treatment of patients with nonhereditary unilateral functional adrenal adenomas. This article is one of the few reports which address the issue of LPA. Their results showed that LPA is safe and feasible, and has similar therapeutic results to laparoscopic total adrenalectomy (LTA) in patients with a nonhereditary hormonally active unilateral adrenal mass [1]. Furthermore, LPA can obviate the need for steroid replacement in these patients [1]. Nakada et al. [2] found that patients with unilateral adrenalectomy do not respond as robustly to stressful situations as do normal controls. Furthermore, if the remaining adrenal gland requires future surgical therapy or suffers functional impairment, the patient may lose his/her remaining source of steroid production [2]. Therefore, preserving the maximal amount of normal adrenal cortex can leave patients with adequate hormonal reserve and avoid the need for long-term steroid therapy. Similar to the authors above, we examined the perioperative surgical and long-term outcomes of LPA [3]. Balci et al. [1] reported that the mean operative time and estimated blood loss were similar between the groups. However, in our study, the mean operative time was significantly shorter and the mean estimated blood loss was significantly higher in the LPA group than in the LTA group. A large and randomized prospective study is required to clarify these different findings. Nevertheless, we believe that LPA has major advantage for preservation of functioning adrenal gland compared to those of LTA. Furthermore, LPA is more meaningful in patients with bilateral involvement than in those with a solitary adrenal gland, as well as those with hereditary adrenal diseases such as multiple endocrine neoplasia and von HippelLandau disease.

\section{Disclosure Statement}

The authors have no conflicts of interest to declare.

\section{Funding Sources}

The authors did not receive any funding.

\section{Author Contributions}

Conception: Y.B. Jeong and Y.S. Shin, drafting: Y.S. Shin, editing and supervision: Y.B. Jeong and H.J. Kim, and final approval: Y.B. Jeong, Y.S. Shin, and H.J. Kim. karger@karger.com www.karger.com/uin

(C) 2020 S. Karger AG, Base

Karger!
Yu Seob Shin

Department of Urology

Jeonbuk National University Medical School

20 Geonji-ro, Jeonju 560-180 (Republic of Korea)

ball1210@hanmail.net 


\section{References}

1 Balci M, Tuncel A, Aslan Y, Aykanat C, Berker D, Guzel O. Laparoscopic partial versus total adrenalectomy in nonhereditary unilateral adrenal masses. Urol Int. 2020;104(12):75-80.

2 Nakada T, Kubota Y, Sasagawa I, Yagisawa T, Watanabe M, Ishigooka M. Therapeutic out- come of primary aldosteronism: adrenalectomy versus enucleation of aldosterone-producing adenoma. J Urol. 1995;153(6):1775-80.

3 Ko OS, Kim JY, Kim HJ, Jeong YB. Laparoscopic partial adrenalectomy: surgical technique and outcome. Korean J Urol Oncol. 2019;17(2):103-9. 